UdeM-GPP-TH-14-234

\title{
Detecting New Physics in Rare Top Decays at the LHC
}

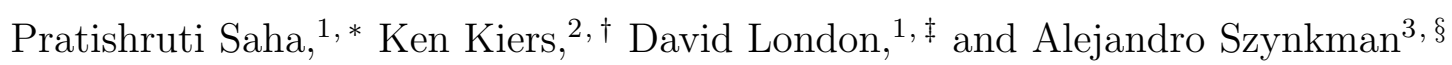 \\ ${ }^{1}$ Physique des Particules, Université de Montréal, \\ C.P. 6128, succ. centre-ville, Montréal, QC, Canada H3C 3J7 \\ ${ }^{2}$ Physics and Engineering Department, Taylor University, \\ 236 West Reade Ave., Upland, IN 46989, USA \\ ${ }^{3}$ IFLP, CONICET - Dpto. de Física, Universidad \\ Nacional de La Plata, C.C. 67, 1900 La Plata, Argentina
}

(Dated: July 8, 2014)

\begin{abstract}
In the companion paper it was shown that there are six observables in $g g \rightarrow t \bar{t} \rightarrow$ $(b \bar{b} c)(\bar{b} \ell \bar{\nu})$ that can be used to reveal the presence of new physics (NP) in $t \rightarrow b \bar{b} c$. In the present paper we examine the prospects for detecting and identifying such NP at the LHC, in both the short term and long term. To this end, we develop an algorithm for extracting the NP parameters from measurements of the observables. In the short term, depending on what measurements have been made, there are several different ways of detecting the presence of NP. It may even be possible to approximately determine the values of certain NP parameters. In the long term, it is expected that all six observables will be measured. The values of the NP parameters can then be determined reasonably precisely from a fit to these measurements, which will provide good information about the type of NP present in $t \rightarrow b \bar{b} c$.
\end{abstract}

PACS numbers: $14.65 . \mathrm{Ha}$

\footnotetext{
*Electronic address: pratishruti.saha@umontreal.ca

${ }^{\dagger}$ Electronic address: knkiers@taylor.edu

${ }^{\ddagger}$ Electronic address: london@lps.umontreal.ca

§Electronic address: szynkman@fisica.unlp.edu.ar
} 


\section{INTRODUCTION}

Top physics provides a fertile ground for new-physics (NP) searches. With a mass close to the electroweak scale, the top quark may well be sensitive to interactions that do not affect other fermions. In Ref. [1], the companion paper, top decay is investigated for the presence of NP. It is noted that, given the good agreement between the experimental measurement of $\Gamma_{t}$ and its theoretical prediction [2], significant NP contributions to top decay can only be present in decay modes that are suppressed in the standard model (SM). One example is $t \rightarrow b \bar{b} c$, whose amplitude involves the small element $V_{c b}(\simeq 0.04)$ of the Cabibbo-Kobayashi-Maskawa (CKM) quark mixing matrix. Ref. [1] focuses on this decay at the LHC, where top production occurs predominantly via gluon fusion: $g g \rightarrow t \bar{t}$. The goal is to find observables in the channel $g g \rightarrow t \bar{t}$ with $t \rightarrow b \bar{b} c$ and $\bar{t} \rightarrow \bar{b} \ell \bar{\nu}$ that can reveal the presence of NP.

NP contributions to the decay $t \rightarrow b \bar{b} c$ can be parametrized in terms of higherdimensional operators. If one restricts to dimension- 6 operators, then this is realized in the form of ten operators that span all possible Lorentz structures. In Ref. [1], two types of observables are identified that can then be used to get a handle on this NP. The first consists of invariant mass-squared distributions involving the $\{b, c\},\{\bar{b}, c\}$, or $\{b, \bar{b}\}$ quark pairs coming from $t \rightarrow b \bar{b} c$. As for the second type, we note that, in $g g \rightarrow t \bar{t}$, the spins of the $t$ and $\bar{t}$ are correlated. The spin-correlation coeffcient $\left(\kappa_{t \bar{t}}\right)$ depends only on the production process. However, since the $t$ quark has an extremely short lifetime, this quantity has to be inferred by measuring the angular correlation between the decay products of the $t$ and those of the $\bar{t}$. If there are NP contributions to the decay, the inferred value of $\kappa_{t \bar{t}}$ is necessarily altered from that of the SM. It is this feature that provides information about the NP. Therefore, the second type of observable consists of these angular correlations. They are taken between the $\ell^{-}$coming from the $\bar{t}$ decay and one of $\bar{b}, b$ or $c$ coming from the $t$ decay.

The NP operators not only change the top branching fraction of this decay, but also modify the shapes of these distributions. It is shown in Ref. [1] that the NP contributions to all of the above observables can be written in terms of certain combinations of the NP couplings, denoted as $\hat{A}_{i}^{\sigma}$. Furthermore, the observables are found to be practically unaffected by parton densities, etc., so that they provide direct access to the values of these $\hat{A}_{i}^{\sigma}$ 's.

Now, the observables described above involve the $\bar{b}$ quark coming from the decay of the $t$. However, there is also a $\bar{b}$ produced in the $\bar{t}$ decay. A realistic analysis must deal with the question of how to distinguish the two $\bar{b}$ 's. In addition, while the focus in Ref. [1] was entirely on $t \bar{t}$ production from gluon fusion, there is also a contribution from $q \bar{q} \rightarrow t \bar{t}$ which must be considered.

In the present paper we address these issues. In Ref. [1], the analytical expressions for the observables are compared with the results of a numerical simulation of the LHC using MadGraph 5 [3]. Here we extend our MadGraph 5 simulations to examine different strategies for extracting the NP parameters. In so doing, we include a method for distinguishing the two $\bar{b}$ 's. We also take the contribution from 
$q \bar{q} \rightarrow t \bar{t}$ into account, examining its effect on the aforementioned observables and their sensitivity to the $\hat{A}_{i}^{\sigma}$ 's. In our simulations we consider numbers of total events representative of LHC measurements in both the short and long terms. While the long-term results obviously have smaller errors, it is still possible in the short term to detect and partially identify NP in $t \rightarrow b \bar{b} c$.

We begin in Sec. II by summarizing the results of Ref. [1]. We present the NP operators that contribute to $t \rightarrow b \bar{b} c$, as well as the $g g \rightarrow t \bar{t} \rightarrow(b \bar{b} c)(\bar{b} \ell \bar{\nu})$ observables that can reveal the presence of the NP. In Sec. III we develop the algorithm to extract the NP parameters from the observables. We discuss the MadGraph 5 simulations in Sec. IV, and apply the algorithm. Here we show that the measurement of the observables at the LHC can lead to the detection of the NP, and possibly even its identification ${ }^{1}$. We conclude in Sec. V.

\section{NEW PHYSICS IN TOP DECAY}

In this section, we summarize the main results of Ref. [1].

\section{A. $t \rightarrow b \bar{b} c:$ effective Lagrangian}

In the SM, the decay $t \rightarrow b \bar{b} c$ proceeds through $t \rightarrow W^{+} b$, followed by $W^{+} \rightarrow \bar{b} c$. The NP contributions to this can be parameterized by the effective Lagrangian $\mathcal{L}_{\text {eff }}=\mathcal{L}_{\text {eff }}^{V}+\mathcal{L}_{\text {eff }}^{S}+\mathcal{L}_{\text {eff }}^{T}$, with

$$
\begin{aligned}
\mathcal{L}_{\text {eff }}^{V}=4 \sqrt{2} G_{F} V_{c b} V_{t b} & \left\{X_{L L}^{V} \bar{b} \gamma_{\mu} P_{L} t \bar{c} \gamma^{\mu} P_{L} b+X_{L R}^{V} \bar{b} \gamma_{\mu} P_{L} t \bar{c} \gamma^{\mu} P_{R} b\right. \\
& \left.+X_{R L}^{V} \bar{b} \gamma_{\mu} P_{R} t \bar{c} \gamma^{\mu} P_{L} b+X_{R R}^{V} \bar{b} \gamma_{\mu} P_{R} t \bar{c} \gamma^{\mu} P_{R} b\right\}+ \text { h.c. }, \\
\mathcal{L}_{\text {eff }}^{S}=4 \sqrt{2} G_{F} V_{c b} V_{t b} & \left\{X_{L L}^{S} \bar{b} P_{L} t \bar{c} P_{L} b+X_{L R}^{S} \bar{b} P_{L} t \bar{c} P_{R} b\right. \\
+ & \left.X_{R L}^{S} \bar{b} P_{R} t \bar{c} P_{L} b+X_{R R}^{S} \bar{b} P_{R} t \bar{c} P_{R} b\right\}+ \text { h.c., } \\
\mathcal{L}_{\text {eff }}^{T}=4 \sqrt{2} G_{F} V_{c b} V_{t b} & \left\{X_{L L}^{T} \bar{b} \sigma^{\mu \nu} P_{L} t \bar{c} \sigma_{\mu \nu} P_{L} b\right. \\
& \left.+X_{R R}^{T} \bar{b} \sigma^{\mu \nu} P_{R} t \bar{c} \sigma_{\mu \nu} P_{R} b\right\}+ \text { h.c. }
\end{aligned}
$$

Here the colour indices are assumed to contract in the same way as in the SM (i.e., the fields $\bar{b}$ with $t$ and $\bar{c}$ with $b$ ). Colour-mismatched terms, in which the indices contract in the opposite way, may occur in certain models and can be incorporated in a straightforward manner [4].

\footnotetext{
${ }^{1}$ When we refer to the "identification of NP", what is implied is the measurement of the various $\hat{A}_{i}^{\sigma}$ 's and $\operatorname{Re}\left(X_{L L}^{V}\right)$.
} 
The NP couplings (the $X_{A B}^{I}$ in the above equations) contain weak phases, but the strong phases are negligible [5]. In addition, the $X_{A B}^{I}$ may be assumed, quite generally, to be $O(1)$. The sizes of the SM and NP contributions to $t \rightarrow b \bar{b} c$ would then be roughly equal. This shows that it is important to include both the SM-NP and NP-NP interference pieces when computing the effect of NP on a particular observable.

\section{B. Observables in $g g \rightarrow t \bar{t} \rightarrow(b \bar{b} c)(\bar{b} \ell \bar{\nu})$}

The kinematics of $g g \rightarrow t \bar{t} \rightarrow(b \bar{b} c)(\bar{b} \ell \bar{\nu})$ is represented in Fig. 1. The six-body phase space is decomposed into five solid angles $d \Omega_{1}^{* *}, d \Omega_{2}^{*}, d \Omega_{4}^{* *}, d \Omega_{5}^{*}$ and $d \Omega_{t}$, and two invariant masses $M_{2}$ and $M_{5}$. The $*$ and $* *$ superscripts on the solid angles indicate that these angles are defined in reference frames that are, respectively, one and two boosts away from the $t \bar{t}$ rest frame. $M_{2}$ and $M_{5}$ are defined by $M_{2}^{2}=$ $\left(p_{1}+p_{2}\right)^{2}$ and $M_{5}^{2}=\left(p_{4}+p_{5}\right)^{2}$. Note that $p_{1}, p_{2}$ and $p_{3}$ are the momenta of the $b$, $\bar{b}$ and $c$ quarks in $t \rightarrow b \bar{b} c$, but all permutations are allowed. The observables use several of these possibilities.

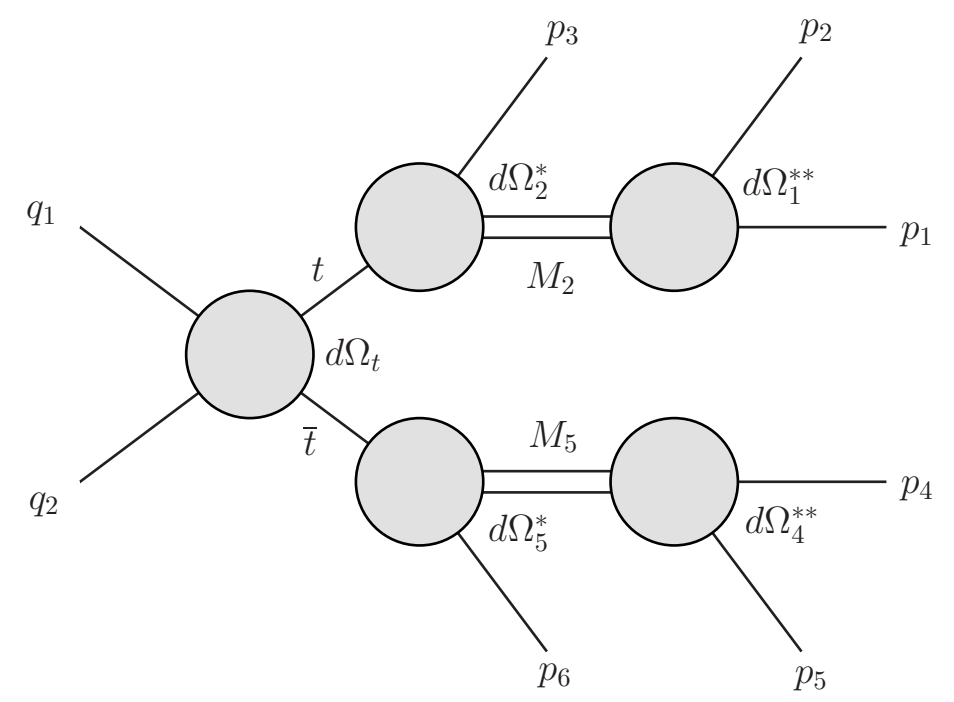

FIG. 1: Kinematics for the process $g g \rightarrow t \bar{t} \rightarrow(b \bar{b} c)(\bar{b} \ell \bar{\nu})[\underline{6}] . \Omega_{1}^{* *}$ denotes the direction of $\vec{p}_{1}{ }^{* *}$ in the rest frame of $M_{2}$, relative to the direction of $\vec{p}_{1}{ }^{*}+\vec{p}_{2}{ }^{*}$, where $M_{2}^{2}=\left(p_{1}+p_{2}\right)^{2}$. Similarly, $\Omega_{2}^{*}$ denotes the direction of $\left(\vec{p}_{1}{ }^{*}+\vec{p}_{2}{ }^{*}\right)$ in the $t$ rest frame, relative to the direction of $\vec{p}_{t}$ in the $t \bar{t}$ rest frame. $\Omega_{t}$ denotes the direction of $\vec{p}_{t}$ relative to $\vec{q}_{1}$, also in the $t \bar{t}$ rest frame. The solid angles $\Omega_{4}^{* *}$ and $\Omega_{5}^{*}$ are defined analogously to $\Omega_{1}^{* *}$ and $\Omega_{2}^{*}$, respectively, and $M_{5}^{2}=\left(p_{4}+p_{5}\right)^{2}$.

The differential cross section for $g g \rightarrow t \bar{t} \rightarrow(b \bar{b} c)(\bar{b} \ell \bar{\nu})$ is computed in Ref. [1]. It is a function of the final-state momenta $p_{i}(i=1,2, . ., 6)$ and SM and NP couplings, 
and is defined with respect to $d M_{2}^{2} d M_{5}^{2} d \Omega_{1}^{* *} d \Omega_{2}^{*} d \Omega_{4}^{* *} d \Omega_{5}^{*} d \Omega_{t}$. The cross section is then integrated over $M_{5}^{2}$ and over all angles except for $\theta_{2}^{*}$ and $\theta_{\ell}^{*}$. The observables are obtained by (i) assigning the $p_{i}$ to specific final-state particles, and (ii) integrating further over $\theta_{2}^{*}$ and $\theta_{\ell}^{*}$, or $M_{2}^{2}$.

There are three possibilities for the particle assignments: (i) $p_{1}=p_{c}, p_{2}=p_{b}$, $p_{3}=p_{\bar{b}_{1}}$, (ii) $p_{1}=p_{c}, p_{2}=p_{\bar{b}_{1}}, p_{3}=p_{b}$, (iii) $p_{1}=p_{b}, p_{2}=p_{\bar{b}_{1}}, p_{3}=p_{c}$. Here

$p_{\bar{b}_{1}}$ refers to the $\bar{b}$ coming from the $t$. Also, $p_{6}=p_{\ell^{-}}$. For each case there are two observables:

\section{Invariant mass-squared distribution:}

$$
\begin{aligned}
\frac{d \sigma}{d \zeta_{12}^{2}}=\sigma_{\mathrm{SM}}\left\{F_{12}\right. & \frac{6 h_{\mathrm{SM}}^{12}\left(\zeta_{12}^{2}\right)}{\left(1-\zeta_{W}^{2}\right)^{2}\left(1+2 \zeta_{W}^{2}\right)} \\
& \left.\quad+\frac{3 G_{F} m_{t}^{2}}{\sqrt{2} \pi^{2}\left(1-\zeta_{W}^{2}\right)^{2}\left(1+2 \zeta_{W}^{2}\right)} \sum_{i, \sigma} \hat{A}_{i}^{\sigma} h_{i}^{12}\left(\zeta_{12}^{2}\right)\right\}
\end{aligned}
$$

\section{Angular correlation:}

$$
\begin{aligned}
\frac{d \sigma}{d \cos \theta_{3}^{*} d \cos \theta_{\ell}^{*}} & =\frac{\sigma_{\mathrm{SM}}}{4}\left\{\left[1+\rho_{3}\left(\zeta_{W}^{2}\right) \kappa(r) \cos \theta_{3}^{*} \cos \theta_{\ell}^{*}\right]\right. \\
& +\frac{3 G_{F} m_{t}^{2}}{4 \sqrt{2} \pi^{2}\left(1-\zeta_{W}^{2}\right)^{2}\left(1+2 \zeta_{W}^{2}\right)}\left[\left(\sum_{i, \sigma} \hat{A}_{i}^{\sigma}\right)\right. \\
& \left.\left.+\left(\hat{A}_{3}^{+}-\hat{A}_{3}^{-}-\frac{1}{3}\left(\hat{A}_{1}^{+}-\hat{A}_{1}^{-}+\hat{A}_{2}^{+}-\hat{A}_{2}^{-}\right)\right) \kappa(r) \cos \theta_{3}^{*} \cos \theta_{\ell}^{*}\right]\right\}
\end{aligned}
$$

The numerical subscripts and superscripts correspond to the particle assignments in each of the three cases. That is, in case (i), the subscript 12 corresponds to $b c(b$ is particle 1, $c$ is particle 2), and similarly for cases (ii) $(12=\bar{b} c)$ and (iii) $(12=b \bar{b})$. In the summations, $\sigma=+,-$ and $i=b, \bar{b}, c$.

$\sigma_{S M}$ is given in Eq. (60) of the Appendix of Ref. [1], $\zeta_{12}^{2} \equiv\left(p_{1}+p_{2}\right)^{2} / m_{t}^{2}, \zeta_{W} \equiv$ $m_{W} / m_{t}$, and $\kappa(r)$ is defined as

$$
\kappa(r)=\frac{\left(-31 r^{4}+37 r^{2}-66\right) r-2\left(r^{6}-17 r^{4}+33 r^{2}-33\right) \tanh ^{-1}(r)}{r^{2}\left[\left(31 r^{2}-59\right) r+2\left(r^{4}-18 r^{2}+33\right) \tanh ^{-1}(r)\right]},
$$

where

$$
r \equiv \sqrt{1-4 m_{t}^{2} / Q^{2}}, Q \equiv p_{t}+p_{\bar{t}} .
$$

The functions $h_{i}^{m n}(m n=b c, \bar{b} c, b \bar{b} ; i=b, \bar{b}, c)$ are defined in Table 【, and

$$
\begin{aligned}
h_{\mathrm{SM}}^{b c}\left(\zeta_{b c}^{2}\right) & =\left(1-\zeta_{b c}^{2}\right) \zeta_{b c}^{2} \theta\left(1-\zeta_{W}^{2}-\zeta_{b c}^{2}\right) \\
h_{\mathrm{SM}}^{\bar{b} c}\left(\zeta_{\bar{b} c}^{2}\right) & =\left(\frac{\zeta_{W} \gamma_{W}}{6 \pi}\right) \frac{\left(1-\zeta_{\bar{b} c}^{2}\right)^{2}\left(1+2 \zeta_{\bar{b} c}^{2}\right)}{\left(\zeta_{\bar{b} c}^{2}-\zeta_{W}^{2}\right)^{2}+\left(\zeta_{W} \gamma_{W}\right)^{2}} \\
h_{\mathrm{SM}}^{b \bar{b}}\left(\zeta_{b \bar{b}}^{2}\right) & =\left(1-\zeta_{W}^{2}-\zeta_{b \bar{b}}^{2}\right)\left(\zeta_{W}^{2}+\zeta_{b \bar{b}}^{2}\right) \theta\left(1-\zeta_{W}^{2}-\zeta_{b \bar{b}}^{2}\right)
\end{aligned}
$$


TABLE I: Definitions of the $h_{i}^{m n}(m n=b c, \bar{b} c, b \bar{b})$ functions. The columns correspond to $i=b, \bar{b}, c$.

\begin{tabular}{c|ccc}
\hline \hline & $b$ & $\bar{b}$ & $c$ \\
\hline$h_{i}^{b c}\left(\zeta^{2}\right)$ & $\frac{1}{2}\left(1-\zeta^{2}\right)^{2}\left(1+2 \zeta^{2}\right)$ & $3\left(1-\zeta^{2}\right)^{2} \zeta^{2}$ & $\frac{1}{2}\left(1-\zeta^{2}\right)^{2}\left(1+2 \zeta^{2}\right)$ \\
\hline$h_{i}^{\bar{b} c}\left(\zeta^{2}\right)$ & $3\left(1-\zeta^{2}\right)^{2} \zeta^{2}$ & $\frac{1}{2}\left(1-\zeta^{2}\right)^{2}\left(1+2 \zeta^{2}\right)$ & $\frac{1}{2}\left(1-\zeta^{2}\right)^{2}\left(1+2 \zeta^{2}\right)$ \\
\hline$h_{i}^{b \bar{b}}\left(\zeta^{2}\right)$ & $\frac{1}{2}\left(1-\zeta^{2}\right)^{2}\left(1+2 \zeta^{2}\right)$ & $\frac{1}{2}\left(1-\zeta^{2}\right)^{2}\left(1+2 \zeta^{2}\right)$ & $3\left(1-\zeta^{2}\right)^{2} \zeta^{2}$ \\
\hline \hline
\end{tabular}

In addition,

$$
\begin{gathered}
\rho_{\bar{b}}\left(\zeta_{W}^{2}\right)=1, \quad \rho_{b}\left(\zeta_{W}^{2}\right)=-\left(\frac{1-2 \zeta_{W}^{2}}{1+2 \zeta_{W}^{2}}\right) \\
\rho_{c}\left(\zeta_{W}^{2}\right)=\frac{1-12 \zeta_{W}^{2}+9 \zeta_{W}^{4}+2 \zeta_{W}^{6}-12 \zeta_{W}^{4} \ln \left(\zeta_{W}^{2}\right)}{\left(1-\zeta_{W}^{2}\right)^{2}\left(1+2 \zeta_{W}^{2}\right)} .
\end{gathered}
$$

and

$$
F_{b c}=F_{b \bar{b}}=1, \quad F_{\bar{b} c}=1-4\left(1-\zeta_{\bar{b} c}^{2} / \zeta_{W}^{2}\right) \operatorname{Re}\left(X_{L L}^{V}\right) .
$$

Note that we have neglected some mild dependence on $\operatorname{Re}\left(X_{L L}^{V}\right)$ in the $b c$ and $b \bar{b}$ distributions. This dependence is, however, properly taken into account in the numerical work below.

The NP parameters appear in the observables in the $\hat{A}_{i}^{\sigma}$ 's:

$$
\begin{aligned}
& \hat{A}_{\bar{b}}^{+}=4\left|X_{L L}^{V}\right|^{2}-8 \operatorname{Re}\left(X_{L L}^{T} X_{L L}^{S *}\right)+32\left|X_{L L}^{T}\right|^{2}, \\
& \hat{A}_{\bar{b}}^{-}=4\left|X_{R R}^{V}\right|^{2}-8 \operatorname{Re}\left(X_{R R}^{T} X_{R R}^{S *}\right)+32\left|X_{R R}^{T}\right|^{2}, \\
& \hat{A}_{b}^{+}=\left|X_{L L}^{S}\right|^{2}+\left|X_{L R}^{S}\right|^{2}-16\left|X_{L L}^{T}\right|^{2}, \\
& \hat{A}_{b}^{-}=\left|X_{R R}^{S}\right|^{2}+\left|X_{R L}^{S}\right|^{2}-16\left|X_{R R}^{T}\right|^{2} \\
& \hat{A}_{c}^{+}=4\left|X_{L R}^{V}\right|^{2}+8 \operatorname{Re}\left(X_{L L}^{T} X_{L L}^{S *}\right)+32\left|X_{L L}^{T}\right|^{2}, \\
& \hat{A}_{c}^{-}=4\left|X_{R L}^{V}\right|^{2}+8 \operatorname{Re}\left(X_{R R}^{T} X_{R R}^{S *}\right)+32\left|X_{R R}^{T}\right|^{2} .
\end{aligned}
$$

As pointed out in the introduction, the six observables have different functional dependencies on the $\hat{A}_{i}^{\sigma}$ 's.

\section{EXTRACTING NEW PHYSICS FROM OBSERVABLES}

In the companion paper [1], we showed that the chosen observables, namely the three invariant mass-squared distributions $\left(d \sigma / d \zeta_{12}^{2}\right)$ and the three angular correlations $\left(d \sigma / d \cos \theta_{3}^{*} \cos \theta_{\ell}^{*}\right)$, are sensitive to certain combinations of the new-physics parameters. This dependence is represented in Eqs. (4) and (5) as combinations of the various $\hat{A}_{i}^{\sigma}$ 's, which in turn can be defined [Eq. (111)] in terms of the NP coefficients $X_{A B}^{I}$ that appear in the effective Lagrangian. In addition, the NP operator 
proportional to $X_{L L}^{V}$ has the same Lorentz structure as the corresponding SM operator, so that these two interfere, leading to an explicit dependence on $X_{L L}^{V}$ itself. As discussed in Ref. [1], the shapes of the observables are largely insensitive to effects due to parton distribution functions (PDFs). It is only natural then to expect that it should be possible to extract the various combinations of NP parameters by fitting these distributions. Further, one expects that, by combining information from all six observables, it should be possible to extract the values of the individual $\hat{A}_{i}^{\sigma}$ 's and $\operatorname{Re}\left(X_{L L}^{V}\right)$ as well. In this section we develop the procedure to carry out this extraction.

\section{A. Algorithm}

Consider first the conventional fitting method, which involves individual observables. If the underlying theory has $N$ theoretical unknowns, their values can be determined only if at least $N$ observables are measured. There are theoretical expressions for these observables in terms of the $N$ unknowns. Using these expressions, the best-fit values of the unknowns are those for which the measured values of the observables are best reproduced.

In our case, the observables are distributions and correlations, specifically $d \sigma / d \zeta_{12}^{2}$ [Eq. (4)] and $d \sigma / d \cos \theta_{3}^{*} \cos \theta_{\ell}^{*}$ [Eq. (5)]. Each distribution/correlation contains many measurements (at different values of $\zeta_{12}^{2}$ or $\left.\left(\cos \theta_{3}^{*}, \cos \theta_{\ell}^{*}\right)\right)$. Equations (4) and (5) show that each of the $\zeta_{12}^{2}$ distributions and the angular correlations can be written as a linear combination of the SM piece and several NP pieces $\left[\operatorname{six} \hat{A}_{i}^{\sigma}\right.$ 's, $\left.\operatorname{Re}\left(X_{L L}^{V}\right)\right]$. With this in mind, we use MadGraph 5 in conjunction with FeynRules [7] to generate eight templates for each distribution/correlation. The templates are nothing but the said distributions/correlations generated with the input values of the parameters chosen such that certain specific contributions to the observables are retained, while all others are set to zero. The objective is to isolate the contributions coming from the SM, the individual $\hat{A}_{i}^{\sigma}$ 's and $\operatorname{Re}\left(X_{L L}^{V}\right)$. Table $\amalg$ gives the parameter choices made for each template (labelled TM-i) and the contributions that they represent.

Once we have these templates, a $\zeta_{12}^{2}$ distribution or an angular correlation arising from a generic choice of NP parameters can be represented as a linear combination of the corresponding templates with appropriate coefficients. Extracting these coefficients allows one to determine the values of the NP parameters involved.

\section{B. Testing the algorithm}

We use "pseudo-data" generated in Monte Carlo simulations to test our fitting procedure. Once again, we use MadGraph 5 to generate these samples. In Ref. [1] we presented plots of the normalized distributions and correlations. Here we use the unnormalized distributions for the fitting. Since both the templates and the 
TABLE II: NP parameter choices for each of the templates TM-i.

\begin{tabular}{|c|c|c|c|}
\hline Template & $X_{A B}^{I}$ & $\hat{A}_{i}^{\sigma}$ & Description \\
\hline $\mathrm{TM}-0$ & All $X_{A B}^{I}=0$ & All $\hat{A}_{i}^{\sigma}=0$ & SM contribution \\
\hline $\mathrm{TM}-1$ & $X_{L L}^{S}, X_{L L}^{T} \neq 0$ & $\hat{A}_{\bar{b}}^{+} \neq 0 ;$ all other $\hat{A}_{i}^{\sigma}=0$ & Contribution $\propto \hat{A}_{\bar{b}}^{+}$ \\
\hline $\mathrm{TM}-2$ & $X_{R R}^{V} \neq 0$ & $\hat{A}_{\bar{b}}^{-} \neq 0 ;$ all other $\hat{A}_{i}^{\sigma}=0$ & Contribution $\propto \hat{A}_{\bar{b}}^{-}$ \\
\hline $\mathrm{TM}-3$ & $X_{L L}^{S} \neq 0$ & $\hat{A}_{b}^{+} \neq 0 ;$ all other $\hat{A}_{i}^{\sigma}=0$ & Contribution $\propto \hat{A}_{b}^{+}$ \\
\hline $\mathrm{TM}-4$ & $X_{R R}^{S} \neq 0$ & $\hat{A}_{b}^{-} \neq 0 ;$ all other $\hat{A}_{i}^{\sigma}=0$ & Contribution $\propto \hat{A}_{b}^{-}$ \\
\hline TM-5 & $X_{L R}^{V} \neq 0$ & $\hat{A}_{c}^{+} \neq 0 ;$ all other $\hat{A}_{i}^{\sigma}=0$ & Contribution $\propto \hat{A}_{c}^{+}$ \\
\hline TM-6 & $X_{R L}^{V} \neq 0$ & $\hat{A}_{c}^{-} \neq 0 ;$ all other $\hat{A}_{i}^{\sigma}=0$ & Contribution $\propto \hat{A}_{c}^{-}$ \\
\hline $\mathrm{TM}-7$ & $X_{L L}^{V} \neq 0$ & $\hat{A}_{\bar{b}}^{+} \neq 0 ;$ all other $\hat{A}_{i}^{\sigma}=0$ & Contributions $\propto \operatorname{Re}\left(X_{L L}^{V}\right)$ and $\hat{A}_{\bar{b}}^{+}$ \\
\hline $\mathrm{TM}-8$ & \multicolumn{2}{|c|}{$\mathrm{TM}-7-\mathrm{TM}-1$} & Contribution $\propto \operatorname{Re}\left(X_{L L}^{V}\right)$ \\
\hline
\end{tabular}

"data-sets" are obtained with MadGraph 5 with the same choices of PDFs, scale, etc., the overall normalization is automatically accounted for.

Our procedure is as follows. We generate pseudo-data using MadGraph 5, in conjunction with FeynRules, for certain chosen values of the NP parameters (i.e., the $\left.X_{A B}^{I}\right)$. This gives us three $\zeta_{12}^{2}$ distributions and three angular correlations. We divide each of these into 25 bins (using a $5 \times 5$ array for the angular correlations). We then perform a single $\chi^{2}$ minimization involving all six histograms in order to determine the coefficients for the templates that result in the best fit for all six observables simultaneously. For this purpose, we use standard, publicly-available routines [8]. Finally, we examine to what extent the values of the NP parameters extracted from the fit agree with their input values.

We consider four different test cases of pseudo-data, which we label EX-1, EX-2, EX-3 and EX-4. The values of the input NP parameters for these test cases are listed in Table III, along with the size of the cross section relative to the SM prediction. The data sets EX-i have been generated for the process $g g \rightarrow t \bar{t} \rightarrow(b \bar{b} c)\left(\bar{b} e^{-} \bar{\nu}_{e}\right)$, taking a benchmark luminosity that corresponds to $10^{5} \mathrm{SM}$ events. The uncertainties incorporated in the fitting procedure are statistical only, and are estimated by considering the number of events in each bin in the histograms to be Poissondistributed. The templates TM-i have been generated for the same process but with $\mathcal{O}\left(10^{6}\right)$ events, so that uncertainties from these can be neglected in the fit. 
TABLE III: Input values of the NP parameters for the four test cases EX-i. The last column illustrates how the total cross section $\sigma$ is affected in each of the test cases.

\begin{tabular}{|c|l|c|c|}
\hline Test Case & \multicolumn{1}{|c|}{$X_{A B}^{I}$} & $\hat{A}_{i}^{\sigma}$ & $\sigma / \sigma_{S M}$ \\
\hline \hline EX-1 & $X_{L L}^{T}=1 ; X_{R R}^{T}=1$ & $\begin{array}{l}\hat{A}_{\bar{b}}^{+}=32 ; \hat{A}_{b}^{+}=-16 ; \hat{A}_{c}^{+}=32 ; \\
\hat{A}_{\bar{b}}^{-}=32 ; \hat{A}_{b}^{-}=-16 ; \hat{A}_{c}^{-}=32\end{array}$ & 3.1 \\
\hline $\mathrm{EX}-2$ & $X_{L R}^{S}=5$ & $\hat{A}_{b}^{+}=25 ;$ all other $\hat{A}_{i}^{\sigma}$ 's $=0$ & 1.6 \\
\hline $\mathrm{EX}-3$ & $X_{L R}^{S}=3 ; X_{R L}^{S}=4$ & $\hat{A}_{b}^{+}=9 ; \hat{A}_{b}^{-}=16 ;$ all other $\hat{A}_{i}^{\sigma}$ 's $=0$ & 1.6 \\
\hline $\mathrm{EX}-4$ & $X_{L L}^{V}=3 ; X_{L L}^{S}=5$ & $\hat{A}_{\bar{b}}^{+}=36 ; \hat{A}_{b}^{+}=25 ;$ all other $\hat{A}_{i}^{\sigma}$ 's $=0$ & 2.4 \\
\hline
\end{tabular}

\section{Fit 1}

As detailed above, the templates are generated by assuming there is only a single contribution at a time to the distributions/correlations for the process $g g \rightarrow t \bar{t} \rightarrow(b \bar{b} c)\left(\bar{b} e^{-} \bar{\nu}_{e}\right)$. The observables are represented by the analytical expressions in Eqs. (41) and (5). However, these expressions have been derived [1] under the (unrealistic) assumption that the two $\bar{b}$ 's in the final state are distinguishable. In Fit 1, as a first test of the algorithm, we retain this assumption.

Table IV] shows the values of the $\hat{A}_{i}^{\sigma}$ 's and $\operatorname{Re}\left(X_{L L}^{V}\right)$ extracted from the fit for the four different test cases of pseudo-data in Table III. A comparison of the two tables shows that most of the values of the parameters extracted from the fit agree with their input values within $\pm 1 \sigma$. This demonstrates that the fundamental idea of the algorithm, namely fitting using the templates, is sound.

The worst-fitted parameter is $\operatorname{Re}\left(X_{L L}^{V}\right)$ in the case where $\hat{A}_{\bar{b}}^{+}$is nonzero but $\operatorname{Re}\left(X_{L L}^{V}\right)=0$. This poor fit is an artifact of the somewhat simple-minded fitting procedure that we adopt: $\hat{A}_{\bar{b}}^{+}$and $\operatorname{Re}\left(X_{L L}^{V}\right)$ are treated as independent parameters in the fit, despite the fact that they are correlated [see Eq. (11)]. Note that the contribution proportional exclusively to $\operatorname{Re}\left(X_{L L}^{V}\right)$ appears primarily in $d \sigma / d \zeta_{\bar{b} c}^{2} \cdot{ }^{2}$ Even so, the fit performs rather well when $\operatorname{Re}\left(X_{L L}^{V}\right)$ is, in fact, nonzero. On the other hand, not considering $\operatorname{Re}\left(X_{L L}^{V}\right)$ as a fit parameter leads to an overall worsening of the fits. For this reason we retain it in our fitting algorithm, while taking care to avoid drawing any strong conclusions from the extracted value of this parameter.

${ }^{2}$ Based on our theoretical analysis, we expect the $\zeta_{\bar{b} c}^{2}$ distribution to have the most sensitivity to $\operatorname{Re}\left(X_{L L}^{V}\right)$. This expectation is confirmed by an examination of the templates. Having said this, the dependence on $\operatorname{Re}\left(X_{L L}^{V}\right)$ is not completely negligible for the other distributions and correlations, and in our numerical work we include the corresponding template (TM-8) in the fits for all distributions and correlations. 
TABLE IV: Values of the NP parameters extracted from the four test cases EX-i using Fit 1.

\begin{tabular}{|c|c|c|}
\hline Test Case & Fit Results & $\chi^{2} /$ d.o.f. \\
\hline $\mathrm{EX}-1$ & $\begin{array}{ll}\text { SM coeff. }=1.005 \pm 0.003 \\
\hat{A}_{\bar{b}}^{+}=33 \pm 2 & \hat{A}_{\bar{b}}^{-}=30 \pm 2 \\
\hat{A}_{b}^{+}=-16 \pm 2 & \hat{A}_{\bar{b}}^{-}=-15 \pm 2 \\
\hat{A}_{c}^{+}=33 \pm 2 & \hat{A}_{c}^{-}=31 \pm 2 \\
\operatorname{Re}\left(X_{L L}^{V}\right)=0.40 \pm 0.02 & \\
\end{array}$ & 1.30 \\
\hline$E X-2$ & $\begin{array}{ll}\text { SM coeff. }=1.000 \pm 0.002 & \\
\hat{A}_{\bar{b}}^{+}=0 \pm 1 & \hat{A}_{\bar{b}}^{-}=0 \pm 1 \\
\hat{A}_{b}^{+}=24 \pm 1 & \hat{A}_{b}^{-}=1 \pm 1 \\
\hat{A}_{c}^{+}=1 \pm 1 & \hat{A}_{c}^{-}=0 \pm 1 \\
\operatorname{Re}\left(X_{L L}^{V}\right)=0.01 \pm 0.02 & \end{array}$ & 1.21 \\
\hline$E X-3$ & $\begin{array}{ll}\text { SM coeff. }=0.994 \pm 0.002 & \\
\hat{A}_{\bar{b}}^{+}=1 \pm 1 & \hat{A}_{\bar{b}}^{-}=-1 \pm 1 \\
\hat{A}_{b}^{+}=10 \pm 1 & \hat{A}_{b}^{-}=15 \pm 1 \\
\hat{A}_{c}^{+}=0 \pm 1 & \hat{A}_{c}^{-}=0 \pm 1 \\
\operatorname{Re}\left(X_{L L}^{V}\right)=0.02 \pm 0.02 & \end{array}$ & 1.22 \\
\hline$E X-4$ & $\begin{array}{ll}\text { SM coeff. }=1.003 \pm 0.003 \\
\hat{A}_{\bar{b}}^{+}=36 \pm 1 & \hat{A}_{\bar{b}}^{-}=0 \pm 1 \\
\hat{A}_{b}^{+}=25 \pm 1 & \hat{A}_{b}^{-}=-1 \pm 1 \\
\hat{A}_{c}^{+}=1 \pm 1 & \hat{A}_{c}^{-}=-1 \pm 1 \\
\operatorname{Re}\left(X_{L L}^{V}\right)=3.03 \pm 0.01 & \end{array}$ & 1.43 \\
\hline
\end{tabular}

\section{Fit 2}

In Fit 2 we drop the assumption that the two final-state $\bar{b}$ 's are distinguishable. The Monte Carlo pseudo-data (as well as the templates) for the process $g g \rightarrow t \bar{t} \rightarrow$ $(b \bar{b} c)\left(\bar{b} e^{-} \bar{\nu}_{e}\right)$ now includes the amplitudes in which the momenta of the two $\bar{b}$ 's in the final state are exchanged. However, in order to construct the above observables, 
we necessarily need to identify the $\bar{b}$ emerging from the top decay. Hence we must restrict our analysis to regions of phase space where the two $\bar{b}$ 's can effectively be considered to be distinguishable. To do this, we construct the two quantities $m_{1}^{2}=\left(p_{b}+p_{c}+p_{\bar{b}_{1}}\right)^{2}$ and $m_{2}^{2}=\left(p_{b}+p_{c}+p_{\bar{b}_{2}}\right)^{2}$. If both $m_{1}$ and $m_{2}$ lie within the range $m_{t} \pm 15 \Gamma_{t}$, the event is discarded. Otherwise, it is assumed that the $\bar{b}_{i}$ that yields the smaller value of $\left|m_{i}-m_{t}\right|$ comes from the $t$-quark decay. This leads to a loss of about $20 \%$ of the events. This cut also distorts the angular correlation such that it no longer conforms to the familiar $\left(a_{1}+a_{2} \cos \theta_{3}^{*} \cos \theta_{\ell}^{*}\right)$ form, even for the SM. Despite the distortion, the fit can be performed using the same algorithm as long as the the same method of event selection is applied to the pseudo-data as well as the templates.

The results of the fit are presented in Table $\mathrm{V}$ for the four test cases. The agreement between the values of the fitted parameters and their input values is almost as good as in the idealized case (Fit 1): apart from $\operatorname{Re}\left(X_{L L}^{V}\right)$ in $\mathrm{EX}-1$, all values agree within $\pm 1.5 \sigma$. We find that, with the event selection discussed above, despite the resulting loss of statistics, one obtains a slight improvement in the goodness-offit, as can be seen from the smaller values of $\chi^{2} /$ d.o.f. All of this demonstrates that our algorithm continues to hold, even when one imposes a 'cut' to distinguish the two $\bar{b}$ 's in the final state.

TABLE V: Values of the NP parameters extracted from the four test cases EX-i using Fit 2.

\begin{tabular}{|c|lr|c|}
\hline Test Case & \multicolumn{2}{|c|}{ Fit Results } & $\chi^{2}$ d.o.f. \\
\hline \hline EX-1 & SM coeff. $=1.005 \pm 0.004 ;$ & 1.09 \\
& $\hat{A}_{\bar{b}}^{+}=29 \pm 2$ & $; \hat{A}_{\bar{b}}^{-}=33 \pm 2$ & \\
& $\hat{A}_{b}^{+}=-15 \pm 2$ & $; \hat{A}_{b}^{-}=-16 \pm 2$ & \\
& $\hat{A}_{c}^{+}=33 \pm 2$ & $; \hat{A}_{c}^{-}=31 \pm 2$ & \\
& $\operatorname{Re}\left(X_{L L}^{V}\right)=0.40 \pm 0.03$ & \\
\hline \hline \multirow{2}{*}{ EX-2 } & SM coeff. $=0.999 \pm 0.003 ;$ & 1.05 \\
& $\hat{A}_{\bar{b}}^{+}=1 \pm 1$ & $; \hat{A}_{\bar{b}}^{-}=-1 \pm 1$ & \\
& $\hat{A}_{b}^{+}=26 \pm 1$ & $; \hat{A}_{b}^{-}=0 \pm 1$ & \\
& $\hat{A}_{c}^{+}=1 \pm 2$ & $; \hat{A}_{c}^{-}=-2 \pm 2$ & \\
& $\operatorname{Re}\left(X_{L L}^{V}\right)=-0.01 \pm 0.02$ & \\
\hline \hline \multirow{2}{*}{ EX-3 } & SM coeff. $=1.005 \pm 0.003 ;$ & $;$ \\
& $\hat{A}_{\bar{b}}^{+}=-1 \pm 1$ & $; \hat{A}_{\bar{b}}^{-}=1 \pm 1$ & \\
& $\hat{A}_{b}^{+}=10 \pm 1$ & $; \hat{A}_{b}^{-}=15 \pm 1$ & \\
\hline
\end{tabular}


TABLE V - continued

\begin{tabular}{|c|c|c|c|}
\hline \multirow[t]{2}{*}{ Test Case } & \multicolumn{2}{|c|}{ Fit Results } & $\chi^{2} /$ d.o.f. \\
\hline & $\begin{array}{l}\hat{A}_{c}^{+}=0 \pm 2 \\
\operatorname{Re}\left(X_{L L}^{V}\right)=-0.01 \pm 0.02\end{array}$ & $; \hat{A}_{c}^{-}=0 \pm 2$ & \\
\hline$E X-4$ & $\begin{array}{l}\text { SM coeff. }=0.997 \pm 0.00 \\
\hat{A}_{\bar{b}}^{+}=38 \pm 2 \\
\hat{A}_{b}^{+}=23 \pm 2 \\
\hat{A}_{c}^{+}=1 \pm 2 \\
\operatorname{Re}\left(X_{L L}^{V}\right)=2.98 \pm 0.02\end{array}$ & $\begin{array}{l}; \\
; \hat{A}_{\bar{b}}^{-}=-1 \pm 2 \\
; \hat{A}_{b}^{-}=2 \pm 2 \\
; \hat{A}_{c}^{-}=-1 \pm 2\end{array}$ & 1.29 \\
\hline
\end{tabular}

\section{Fit 3}

Finally, at the LHC, there is a small $(\approx 10-15 \%)$ contribution to $t \bar{t}$ production from $q \bar{q}$ annihilation. In Fit 3 we consider the impact of this additional contribution.

It must be said that we do not expect a significant effect. Since the NP couplings play a role only in top decay, the structure of Eqs. (4) and (5) remains largely unchanged. The change in Eq. (4) is the analytical form of the factor $\sigma_{S M}$; in Eq. (5), the changes appear in the expressions for $\sigma_{S M}$ and $\kappa(r)$. The decomposition of the NP contribution in terms of a linear combination of $\hat{A}_{i}^{\sigma}$ 's and $\operatorname{Re}\left(X_{L L}^{V}\right)$ therefore

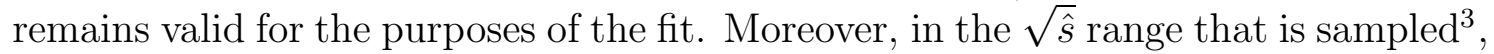
$t \bar{t}$ production is overwhelmingly dominated by $g g$ fusion, simply because the gluon density is large at low values of momentum fractions (the well-known Bjorken $x_{1}$ and $x_{2}$ ). This means that the corrections due to $q \bar{q} \rightarrow t \bar{t}$ are small in magnitude over the entire region of phase space that can be probed.

The results of Fit 3 are presented in Table VI, As expected, the fitting procedure described above proves just as effective for the full process $p p \rightarrow t \bar{t} \rightarrow(b \bar{b} c)\left(\bar{b} e^{-} \bar{\nu}_{e}\right)$.

TABLE VI: Values of the NP parameters extracted from the four test cases EX-i using Fit 3.

\begin{tabular}{|c|r|c|}
\hline Test Case & Fit Results & $\chi^{2} /$ d.o.f. \\
\hline \hline EX-1 & SM coeff. $=1.002 \pm 0.004 ;$ & 1.01 \\
& $\hat{A}_{\bar{b}}^{+}=32 \pm 3 \quad ; \hat{A}_{\bar{b}}^{-}=31 \pm 3$ & \\
\hline
\end{tabular}

\footnotetext{
${ }^{3}$ At a $14 \mathrm{TeV}$ pp collider, with $\mathcal{O}\left(10^{5}\right)$ events, this range is approximately $350 \mathrm{GeV}$ to $1200 \mathrm{GeV}$.
} 
TABLE VI - continued

\begin{tabular}{|c|c|c|c|}
\hline \multirow[t]{2}{*}{ Test Case } & \multicolumn{2}{|c|}{ Fit Results } & \multirow[t]{2}{*}{$\chi^{2} /$ d.o.f. } \\
\hline & $\begin{array}{l}\hat{A}_{b}^{+}=-16 \pm 2 \\
\hat{A}_{c}^{+}=32 \pm 3 \\
\operatorname{Re}\left(X_{L L}^{V}\right)=0.42 \pm 0.03\end{array}$ & $\begin{array}{l}; \hat{A}_{b}^{-}=-15 \pm 2 \\
; \hat{A}_{c}^{-}=33 \pm 3\end{array}$ & \\
\hline$E X-2$ & $\begin{array}{l}\text { SM coeff. }=0.998 \pm 0.003 \\
\hat{A}_{\bar{b}}^{+}=-1 \pm 2 \\
\hat{A}_{b}^{+}=24 \pm 2 \\
\hat{A}_{c}^{+}=1 \pm 2 \\
\operatorname{Re}\left(X_{L L}^{V}\right)=-0.01 \pm 0.02\end{array}$ & $\begin{array}{l}; \hat{A}_{\bar{b}}^{-}=0 \pm 2 \\
; \hat{A}_{b}^{-}=1 \pm 2 \\
; \hat{A}_{c}^{-}=-1 \pm 2\end{array}$ & 1.00 \\
\hline$E X-3$ & $\begin{array}{l}\mathrm{SM} \text { coeff. }=1.001 \pm 0.003 \\
\hat{A}_{\bar{b}}^{+}=1 \pm 2 \\
\hat{A}_{b}^{+}=9 \pm 2 \\
\hat{A}_{c}^{+}=1 \pm 2 \\
\operatorname{Re}\left(X_{L L}^{V}\right)=-0.01 \pm 0.02\end{array}$ & $\begin{array}{l}; \\
; \hat{A}_{\bar{b}}^{-}=-1 \pm 2 \\
; \hat{A}_{b}^{-}=16 \pm 2 \\
; \hat{A}_{c}^{-}=-1 \pm 2\end{array}$ & 1.08 \\
\hline$E X-4$ & $\begin{array}{l}\text { SM coeff. }=0.999 \pm 0.003 \\
\hat{A}_{\bar{b}}^{+}=38 \pm 2 \\
\hat{A}_{b}^{+}=24 \pm 2 \\
\hat{A}_{c}^{+}=1 \pm 3 \\
\operatorname{Re}\left(X_{L L}^{V}\right)=2.97 \pm 0.02\end{array}$ & $\begin{array}{l}; \hat{A}_{\bar{b}}^{-}=-2 \pm 2 \\
; \hat{A}_{b}^{-}=1 \pm 2 \\
; \hat{A}_{c}^{-}=-1 \pm 2\end{array}$ & 1.01 \\
\hline
\end{tabular}

Although the essential structure of our statistical analysis is based on the analytical expressions obtained in Ref. [1], where several simplifying assumptions were made, through the above series of fits we have obtained a reliable algorithm that includes a procedure to distinguish the two final-state $\bar{b}$ 's, and works well even in the presence of the contribution from $q \bar{q} \rightarrow t \bar{t}$. We now use this algorithm to examine the prospects for obtaining information about NP in the decay $t \rightarrow b \bar{b} c$ at the LHC, in both the short and long terms. This is discussed in the next section. 


\section{DETECTING NEW PHYSICS IN TOP DECAY}

Above, we have established a method for the extraction of NP parameters involved in the decay $t \rightarrow b \bar{b} c$. However, should a sizeable NP contribution exist, it is likely that it would first be detected simply by measuring the total cross section in this channel. It is only afterwards that the $\zeta_{12}^{2}$ distributions and the angular correlations discussed in the preceding sections would be used to indicate the presence of NP. While this is true, it should also be pointed out that the overall normalization of the cross section suffers from inherent theoretical uncertainties such as the choice of PDFs, the renormalization and factorization scales, etc. On the other hand, compared to the total cross section, the distributions/correlations have additional discriminating power since their shapes also get modified under the influence of NP.

In the following subsections, using the distributions/correlations, we perform simulations to examine the prospects for detecting NP, for measuring certain combinations of NP parameters, and for partially identifying the NP. The simulations are done using a total number of events consistent with either short-term or long-term measurements at the LHC.

\section{A. Short term}

The $t \bar{t}$ cross section at the LHC at a centre-of-mass energy of $14 \mathrm{TeV}$ is $\sim 900 \mathrm{pb}$ 9]. Considering the SM branching fractions for $t \rightarrow b \bar{b} c$ and $\bar{t} \rightarrow \bar{b} \ell^{-} \bar{\nu}_{\ell}$, the effective cross section in this channel is $\sim 0.1 \mathrm{pb}$. For the short-term simulations, we consider an integrated luminosity which, after factoring in the $b$-tagging efficiency ${ }^{4}$, will lead to $10^{4}$ events of the type $p p \rightarrow t \bar{t} \rightarrow(b \bar{b} c)\left(\bar{b} \ell^{-} \bar{\nu}_{\ell}\right)$ from the SM alone ${ }^{5}$. This is expected to be delivered by 2020-2021 [10].

In the preceding sections, we noted that, in the presence of NP, the shapes of the distributions/correlations can be modified. This suggests that NP can be detected by examining a particular distribution/correlation and seeing a clear difference between the measured shape and its SM prediction. This is explored in Fig. 2. Here all three $\zeta_{12}^{2}$ distributions are shown for the NP scenario EX-1. Clearly, in the cases of $d \sigma / d \zeta_{b c}^{2}$ and $d \sigma / d \zeta_{\bar{b} c}^{2}$, the measurement of the distributions alone would indicate the presence of NP. On the other hand, it would be difficult to draw conclusions from the shape of the corresponding $d \sigma / d \zeta_{b \bar{b}}^{2}$ distribution. ${ }^{6}$

\footnotetext{
${ }^{4}$ This is assumed to be $70 \%$ for each of the three $b$ or $\bar{b}$ 's in the final state

${ }^{5}$ Here $\ell=e, \mu$. In the $\mathrm{CP}$-conserving scenario that we consider, there would be an equal number of events in which $\bar{t} \rightarrow \bar{b} b \bar{c}$ and $t \rightarrow b \ell^{+} \nu$. We assume that the events in which the $\bar{t}$ decays leptonically can be identified by tagging the charge of the lepton and consider only those events in our analysis.

${ }^{6}$ If we were not normalizing the distribution to the total number of events, the difference between the SM and EX-1 cases would be much more apparent.
} 


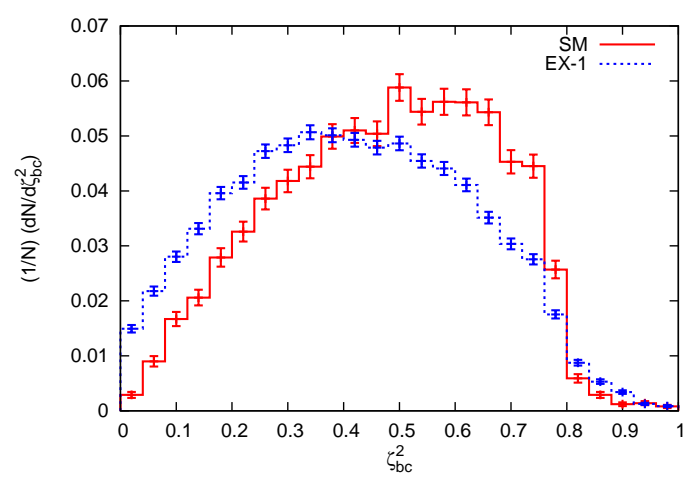

(a)

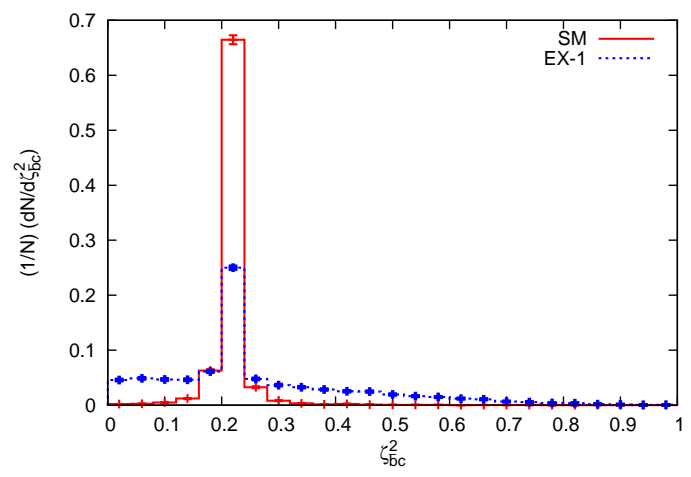

(c)

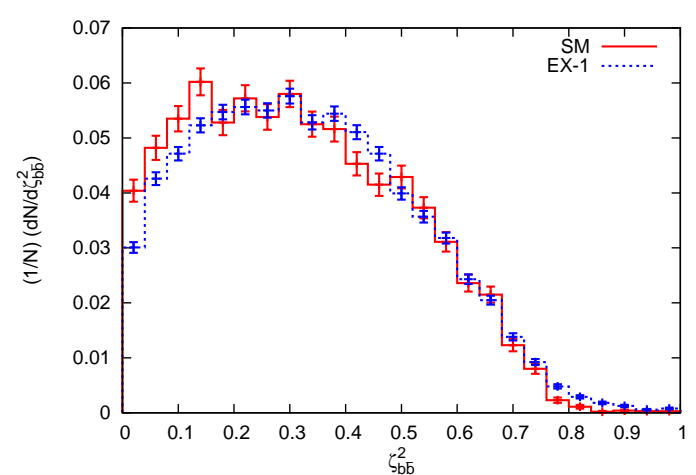

(b)

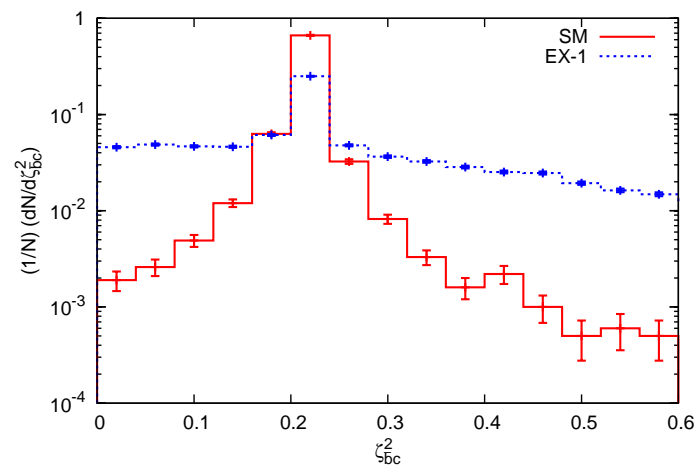

(d)

FIG. 2: Normalized $d \sigma / d \zeta_{12}^{2}$ distributions: (a) $d \sigma / d \zeta_{b c}^{2}$, (b) $d \sigma / d \zeta_{b \bar{b}}^{2}$, (c) $d \sigma / d \zeta_{\bar{b} c}^{2}$, (d) $d \sigma / d \zeta_{\bar{b} c}^{2}$ on a semi-log scale.

However, even in the case of $d \sigma / d \zeta_{b \bar{b}}^{2}$, information about the NP can be obtained. To see this, we use the fitting procedure developed in the previous section and examine what kind of information can be extracted by fitting this distribution alone. From Eq. (4) one sees that the $\zeta_{b \bar{b}}^{2}$ distribution depends on three distinct kinematic structures: $h_{S M}^{b \bar{b}}, h_{c}^{b \bar{b}}$ and $h_{b}^{b \bar{b}}\left(=h_{\bar{b}}^{b \bar{b}}\right)$. These kinematic structures will in principle be modified by cuts, such as we place on the final state $\bar{b}$ 's. In addition, as described above, there is a mild (but potentially important) dependence on $\operatorname{Re}\left(X_{L L}^{V}\right)$ in the $\zeta_{b \bar{b}}^{2}$ distribution. We did not include this dependence in the analytical expressions above, but we do retain it here in our numerical work. Thus, a fit using only the $\zeta_{b \bar{b}}^{2}$ distribution would be sensitive to the relative weights of the SM contribution, $\operatorname{Re}\left(X_{L L}^{V}\right),\left(\hat{A}_{c}^{+}+\hat{A}_{c}^{-}\right)$and $\left(\hat{A}_{\bar{b}}^{+}+\hat{A}_{\bar{b}}^{-}+\hat{A}_{b}^{+}+\hat{A}_{b}^{+}\right)$. Accordingly, we modify our fitting procedure: instead of using all eight templates in the fit, we use only four, namely TM-0, TM-1, TM-5 and TM-8. The values that we obtain for the above combinations of NP parameters are presented in Table VII. Once again, $\operatorname{Re}\left(X_{L L}^{V}\right)$ proves to be the weakest link. For the other combinations of NP parameters, the values extracted from the fit agree with their input values within $\pm 3.2 \sigma$. However, the key point is this: in each test case, a parameter combination whose input value is nonzero is found from the fit to be nonzero to at least $7 \sigma$. So, for the NP scenario EX-1, 
although one cannot draw any conclusions about NP from a visual examination of the normalized $d \sigma / d \zeta_{b \bar{b}}^{2}$ distribution, a fit provides statistically-significant evidence that NP is present.

TABLE VII: Values of the combinations of NP parameters extracted from $d \sigma / d \zeta_{b \bar{b}}^{2}$. The integrated luminosity corresponds to $10^{4} \mathrm{SM}$ events.

\begin{tabular}{|c|c|c|c|c|}
\hline Test Case & Parameter & Input Value & Fit Result & $\chi^{2} /$ d.o.f. \\
\hline$E X-1$ & $\begin{array}{c}\text { SM coefficient } \\
\hat{A}_{c}^{+}+\hat{A}_{c}^{-} \\
\hat{A}_{\bar{b}}^{+}+\hat{A}_{\bar{b}}^{-}+\hat{A}_{b}^{+}+\hat{A}_{b}^{-} \\
\operatorname{Re}\left(X_{L L}^{V}\right)\end{array}$ & $\begin{array}{l}1 \\
64 \\
32 \\
0\end{array}$ & $\begin{array}{c}1.2 \pm 0.2 \\
72 \pm 6 \\
16 \pm 5 \\
3 \pm 1\end{array}$ & 1.31 \\
\hline$E X-2$ & $\begin{array}{c}\text { SM coefficient } \\
\hat{A}_{c}^{+}+\hat{A}_{c}^{-} \\
\hat{A}_{\bar{b}}^{+}+\hat{A}_{\bar{b}}^{-}+\hat{A}_{b}^{+}+\hat{A}_{b}^{-} \\
\operatorname{Re}\left(X_{L L}^{V}\right)\end{array}$ & $\begin{array}{c}1 \\
0 \\
25 \\
0\end{array}$ & $\begin{array}{c}0.9 \pm 0.1 \\
-1 \pm 4 \\
28 \pm 3 \\
0.0 \pm 0.7\end{array}$ & 1.24 \\
\hline$E X-3$ & $\begin{array}{c}\text { SM coefficient } \\
\hat{A}_{c}^{+}+\hat{A}_{c}^{-} \\
\hat{A}_{\bar{b}}^{+}+\hat{A}_{\bar{b}}^{-}+\hat{A}_{b}^{+}+\hat{A}_{b}^{-} \\
\operatorname{Re}\left(X_{L L}^{V}\right) \\
\end{array}$ & $\begin{array}{c}1 \\
0 \\
25 \\
0\end{array}$ & $\begin{array}{c}1.3 \pm 0.1 \\
-10 \pm 4 \\
22 \pm 3 \\
-1.0 \pm 0.7 \\
\end{array}$ & 1.32 \\
\hline $\mathrm{EX}-4$ & $\begin{array}{c}\text { SM coefficient } \\
\hat{A}_{c}^{+}+\hat{A}_{c}^{-} \\
\hat{A}_{\bar{b}}^{+}+\hat{A}_{\bar{b}}^{-}+\hat{A}_{b}^{+}+\hat{A}_{b}^{-} \\
\operatorname{Re}\left(X_{L L}^{V}\right)\end{array}$ & $\begin{array}{l}1 \\
0 \\
61 \\
3\end{array}$ & $\begin{array}{c}1.0 \pm 0.1 \\
-2 \pm 5 \\
63 \pm 4 \\
2.3 \pm 0.8\end{array}$ & 0.96 \\
\hline
\end{tabular}

Similar fits can be performed with the $d \sigma / d \zeta_{b c}^{2}$ and $d \sigma / d \zeta_{\bar{b} c}^{2}$ distributions to extract other combinations of $\hat{A}_{i}^{\sigma}$ 's. If all the $d \sigma / d \zeta_{12}^{2}$ 's are combined in one fit, it is possible to obtain the combinations $\left(\hat{A}_{\bar{b}}^{+}+\hat{A}_{\bar{b}}^{-}\right),\left(\hat{A}_{b}^{+}+\hat{A}_{b}^{-}\right)$and $\left(\hat{A}_{c}^{+}+\hat{A}_{c}^{-}\right)$.

Analogous to the above example, one may wish to attempt the fit of a single angular correlation. This, however, is a more complicated task. As argued earlier, the fits are not sensitive to the individual $\hat{A}_{i}^{\sigma}$ 's, but rather to the different kinematic structures that are present. In the case of the angular correlations [see Eq. (5)], there are two kinematic structures: a constant term and a term proportional to $\cos \theta_{3}^{*} \cos \theta_{\ell}^{*}$. However, the coefficients of these pieces involve both SM and NP 
parameters. Therefore only these combinations of SM and NP parameters can be extracted. Furthermore, note that, in the case of the $\zeta_{12}^{2}$ distributions, each template is proportional to a single kinematic structure. For example, for $d \sigma / d \zeta_{b \bar{b}}^{2}$, TM-5 is only sensitive to $h_{c}^{b \bar{b}}$. On the other hand, in the case of the angular correlations, each template contains both the constant piece and the $\cos \theta_{3}^{*} \cos \theta_{\ell}^{*}$ piece.

In order to work around these difficulties, we proceed as follows. First, we fix the weight of the SM contribution ${ }^{7}$ to be 1.0. Second, the templates themselves have to be reorganized. For example, consider $d \sigma / d \cos \theta_{c}^{*} d \cos \theta_{\ell}^{*}$. Here a template defined as (TM-5 + TM-6) would be proportional to the constant piece, and another defined as (TM-5 - TM-6) would be proportional to the $\cos \theta_{c}^{*} \cos \theta_{\ell}^{*}$ piece $^{8}$. The coefficients of these two modified templates would then be expected to yield the values of $\left(\hat{A}_{c}^{+}+\hat{A}_{c}^{-}+\hat{A}_{\bar{b}}^{+}+\hat{A}_{\bar{b}}^{-}+\hat{A}_{b}^{+}+\hat{A}_{b}^{-}\right)$and $\left(\hat{A}_{c}^{+}-\hat{A}_{c}^{-}-\frac{1}{3}\left\{\hat{A}_{\bar{b}}^{+}-\hat{A}_{\bar{b}}^{-}+\hat{A}_{b}^{+}-\hat{A}_{b}^{-}\right\}\right)$.

The results of the fit for the different EX-i are presented in Table VIII. For all four EX-i, the agreement between best-fit and input values is very good for $\left(\hat{A}_{c}^{+}+\hat{A}_{c}^{-}+\hat{A}_{\bar{b}}^{+}+\hat{A}_{\bar{b}}^{-}+\hat{A}_{b}^{+}+\hat{A}_{b}^{-}\right)$. The key point is that, in all cases, this combination of NP parameters is definitely nonzero. For $\left(\hat{A}_{c}^{+}-\hat{A}_{c}^{-}-\frac{1}{3}\left\{\hat{A}_{\bar{b}}^{+}-\hat{A}_{\bar{b}}^{-}+\hat{A}_{b}^{+}-\hat{A}_{b}^{-}\right\}\right)$the error bars are larger: the best-fit and input values agree to within 1-3 $\sigma$. Nevertheless, a fit to a single angular correlation can provide statistically-significant evidence that NP is present. The measurement of an angular correlation would, however, most likely be more challenging than the measurement of a $\zeta_{12}^{2}$ distribution, which is essentially an invariant mass-squared distribution. Hence it is very likely that NP, if present, will be discovered first in a $\zeta_{12}^{2}$ distribution.

TABLE VIII: Values of the combinations of NP parameters extracted from $d \sigma / d \cos \theta_{c}^{*} d \cos \theta_{\ell}^{*}$. The integrated luminosity corresponds to $10^{4} \mathrm{SM}$ events. The weight of the SM contribution is fixed to be 1.0 .

\begin{tabular}{|c|c|c|c|c|}
\hline Test Case & Parameter & Input Value & Fit Result & $\chi^{2} /$ d.o.f. \\
\hline \hline \multirow{2}{*}{$\mathrm{EX}-1$} & $\hat{A}_{c}^{+}+\hat{A}_{c}^{-}+\hat{A}_{\bar{b}}^{+}+\hat{A}_{\bar{b}}^{-}+\hat{A}_{b}^{+}+\hat{A}_{b}^{-}$ & 96 & $97 \pm 1$ & 1.25 \\
& $\hat{A}_{c}^{+}-\hat{A}_{c}^{-}-\frac{1}{3}\left(\hat{A}_{\bar{b}}^{+}-\hat{A}_{\bar{b}}^{-}+\hat{A}_{b}^{+}-\hat{A}_{b}^{-}\right)$ & 0 & $26 \pm 11$ & \\
\hline \hline $\mathrm{EX}-2$ & $\hat{A}_{c}^{+}+\hat{A}_{c}^{-}+\hat{A}_{\bar{b}}^{+}+\hat{A}_{\bar{b}}^{-}+\hat{A}_{b}^{+}+\hat{A}_{b}^{-}$ & 25 & $26 \pm 1$ & 1.05 \\
& $\hat{A}_{c}^{+}-\hat{A}_{c}^{-}-\frac{1}{3}\left(\hat{A}_{\bar{b}}^{+}-\hat{A}_{\bar{b}}^{-}+\hat{A}_{b}^{+}-\hat{A}_{b}^{-}\right)$ & -8.33 & $-10 \pm 7$ & \\
\hline \hline $\mathrm{EX}-3$ & $\hat{A}_{c}^{+}+\hat{A}_{c}^{-}+\hat{A}_{\bar{b}}^{+}+\hat{A}_{\bar{b}}^{-}+\hat{A}_{b}^{+}+\hat{A}_{b}^{-}$ & 25 & $26 \pm 1$ & 1.06 \\
& $\hat{A}_{c}^{+}-\hat{A}_{c}^{-}-\frac{1}{3}\left(\hat{A}_{\bar{b}}^{+}-\hat{A}_{\bar{b}}^{-}+\hat{A}_{b}^{+}-\hat{A}_{b}^{-}\right)$ & 2.33 & $-3 \pm 7$ & \\
\hline
\end{tabular}

\footnotetext{
${ }^{7}$ Note that this could also have been done for the fits to the $\zeta_{12}^{2}$ distributions. However, in the case of an angular correlation it must be done.

8 This holds as long as the values of $\hat{A}_{c}^{+}$and $\hat{A}_{c}^{-}$used to generate TM-5 and TM-6, respectively, are identical.
} 
TABLE VIII - continued

\begin{tabular}{|c|c|c|c|c|}
\hline Test Case & Parameter & Input Value & Fit Result & $\chi^{2} /$ d.o.f. \\
\hline \hline \multirow{2}{*}{ EX-4 } & $\hat{A}_{c}^{+}+\hat{A}_{c}^{-}+\hat{A}_{\bar{b}}^{+}+\hat{A}_{\bar{b}}^{-}+\hat{A}_{b}^{+}+\hat{A}_{b}^{-}$ & 61 & $64 \pm 1$ & 1.02 \\
& $\hat{A}_{c}^{+}-\hat{A}_{c}^{-}-\frac{1}{3}\left(\hat{A}_{\bar{b}}^{+}-\hat{A}_{\bar{b}}^{-}+\hat{A}_{b}^{+}-\hat{A}_{b}^{-}\right)$ & -20.33 & $-31 \pm 9$ & \\
\hline
\end{tabular}

The simplest approach towards the fitting of the angular correlations would have been to fit them to the functional form $a_{1}+a_{2} \cos \theta_{3}^{*} \cos \theta_{\ell}^{*}$, as is the usual procedure for measuring $\kappa_{t \bar{t}}$. However, this possibility is precluded due to the fact that the event-selection criteria described in Fit 2 distorts the shape of the correlation. We have used a somewhat simpel-minded approach to deal with the identical $\bar{b}$ 's in the final state. It is certainly possible that experimentalists will find a better way to deal with this situation (perhaps through the use of some sophisticated multivariate technique, such as neural networks or boosted decision trees) and that such an approach would lead to less distortion of the shape of the correlation.

Finally, we consider the full fit involving all six observables, with statistics corresponding to $10^{4}$ events for the SM. The results are presented in Table IX. Apart from $\operatorname{Re}\left(X_{L L}^{V}\right)$ in $\mathrm{EX}-1$, the values of all NP parameters agree with their input values within $\pm 1.7 \sigma$.

TABLE IX: Values of the NP parameters extracted from a fit to all six observables. The integrated luminosity corresponds to $10^{4} \mathrm{SM}$ events.

\begin{tabular}{|c|lc|c|}
\hline Test Case & \multicolumn{2}{|c|}{ Fit Results } & $\chi^{2}$ d.o.f. \\
\hline \hline EX-1 & SM coefficient $=1.00 \pm 0.01$ & 1.18 \\
& $\hat{A}_{\bar{b}}^{+}=36 \pm 9$ & $\hat{A}_{\bar{b}}^{-}=28 \pm 9$ & \\
& $\hat{A}_{b}^{+}=-22 \pm 8$ & $\hat{A}_{b}^{-}=-11 \pm 8$ & \\
& $\hat{A}_{c}^{+}=47 \pm 9$ & $\hat{A}_{c}^{-}=18 \pm 9$ & \\
& $\operatorname{Re}\left(X_{L L}^{V}\right)=0.31 \pm 0.09$ & & \\
\hline \hline \multirow{2}{*}{$\mathrm{EX}-2$} & \multicolumn{2}{|c|}{$\mathrm{SM}$ coefficient $=0.988 \pm 0.009$} & 0.92 \\
& $\hat{A}_{\bar{b}}^{+}=6 \pm 6$ & $\hat{A}_{\bar{b}}^{-}=-3 \pm 6$ & \\
& $\hat{A}_{b}^{+}=23 \pm 5$ & $\hat{A}_{b}^{-}=1 \pm 6$ & \\
& $\hat{A}_{c}^{+}=0 \pm 6$ & $\hat{A}_{c}^{-}=-1 \pm 6$ & \\
& $\operatorname{Re}\left(X_{L L}^{V}\right)=0.03 \pm 0.05$ & & \\
\hline \hline \multirow{2}{*}{$\mathrm{EX}-3$} & $\operatorname{SM}$ coefficient $=1.013 \pm 0.009$ & \\
& $\hat{A}_{\bar{b}}^{+}=4 \pm 6$ & $\hat{A}_{\bar{b}}^{-}=-3 \pm 6$ & \\
\hline
\end{tabular}


TABLE IX - continued

\begin{tabular}{|c|lr|c|}
\hline Test Case & \multicolumn{2}{|c|}{ Fit Results } & $\chi^{2} /$ d.o.f. \\
\hline & $\hat{A}_{b}^{+}=16 \pm 5$ & $\hat{A}_{b}^{-}=8 \pm 6$ & \\
& $\hat{A}_{c}^{+}=1 \pm 6$ & $\hat{A}_{c}^{-}=-2 \pm 6$ & \\
& $\operatorname{Re}\left(X_{L L}^{V}\right)=0.03 \pm 0.05$ & & \\
\hline \hline \multirow{2}{*}{$\mathrm{XX}-4$} & $\mathrm{SM}$ coefficient $=1.00 \pm 0.01$ & 0.99 \\
& $\hat{A}_{\bar{b}}^{+}=47 \pm 7$ & $\hat{A}_{\bar{b}}^{-}=-12 \pm 7$ & \\
& $\hat{A}_{b}^{+}=34 \pm 7$ & $\hat{A}_{b}^{-}=-9 \pm 7$ & \\
& $\hat{A}_{c}^{+}=8 \pm 8$ & $\hat{A}_{c}^{-}=-8 \pm 8$ & \\
& $\operatorname{Re}\left(X_{L L}^{V}\right)=2.91 \pm 0.05$ & & \\
\hline
\end{tabular}

To sum up, the above simulations demonstrate that, even in the short term, it is possible to detect the presence of NP in top decay through the measurement of the invariant mass-squared distributions and/or the angular correlations. This can be done by comparing the measured shapes of the distributions/correlations with the SM predictions. More sensitivity can be obtained by performing fits to extract combinations of NP parameters. If all six distributions and correlations can be measured, a combined fit can be performed to extract all the NP parameters. The determination of which parameters are nonzero allows for a partial identification of the NP.

\section{B. Long term}

As noted above, the effective cross section in $g g \rightarrow t \bar{t} \rightarrow(b \bar{b} c)(\bar{b} \ell \bar{\nu})$ is $\sim 0.1$ pb. The LHC is projected to deliver $3000 \mathrm{fb}^{-1}$ worth of data by the year 2030 [10]. Assuming this integrated luminosity and a $b$-tagging efficiency of $70 \%$ for each of the three $b$ or $\bar{b}$ 's in the final state, one obtains $\approx 10^{5}$ events of the type $p p \rightarrow t \bar{t} \rightarrow(b \bar{b} c)\left(\bar{b} \ell^{-} \bar{\nu}_{\ell}\right)$ from the SM. This is the number of events in our long-term simulations.

By 2030, all six distributions and correlations will, in all likelihood, have been measured. For this reason we consider only the fit to all distributions/correlations with $10^{5} \mathrm{SM}$ events. The corresponding results have already been presented in Table VI. Apart from $\operatorname{Re}\left(X_{L L}^{V}\right)$ in EX-1, the best-fit values of all NP parameters differ from their input values by at most $1 \sigma$. The errors on the $\hat{A}_{i}^{\sigma}$ 's are typically in the range $1.75-2.75$. Thus, any $\hat{A}_{i}^{\sigma}$ that is $\gtrsim 10$ will be found to be nonzero at a statistically-significant level. In this way it will be possible to determine which NP parameters are nonzero, thus producing an identification of the NP. 


\section{CONCLUSIONS}

In Ref. [1], the companion paper, new physics (NP) in the decay $t \rightarrow b \bar{b} c$ is considered. There, ten dimension- 6 NP operators contributing to $t \rightarrow b \bar{b} c$ are delineated, and two types of observables are identified that can be used to search for this NP in the process $g g \rightarrow t \bar{t} \rightarrow(b \bar{b} c)(\bar{b} \ell \bar{\nu})$. They are (i) invariant mass-squared distributions involving the $\{b, c\},\{\bar{b}, c\}$, or $\{b, \bar{b}\}$ quark pairs coming from $t \rightarrow b \bar{b} c$, and (ii) angular correlations between the $\ell^{-}$coming from the $\bar{t}$ decay and one of $\bar{b}, b$ or $c$ coming from the $t$ decay. It is further shown that the NP contributions to these observables can be written in terms of certain combinations of the NP couplings, denoted as $\hat{A}_{i}^{\sigma}$. In the present paper we examine the prospects for detecting and identifying such NP at the LHC.

The first step is to develop an algorithm to extract the $\hat{A}_{i}^{\sigma}$ 's and $\operatorname{Re}\left(X_{L L}^{V}\right)$ from the observables. From the analytical expressions obtained in Ref. [1] [summarized here in Eqs. (44) and (5)], we learn that the NP contribution to the observables can be represented as a linear combination of pieces proportional to the different $\hat{A}_{i}^{\sigma}$ 's and $\operatorname{Re}\left(X_{L L}^{V}\right)$. Using this idea, we perform a Monte-Carlo simulation using MadGraph 5 to compute "templates," which are the contributions of the SM, each $\hat{A}_{i}^{\sigma}$ and $\operatorname{Re}\left(X_{L L}^{V}\right)$ to the observables. We also generate Monte-Carlo data for four possible NP scenarios. For each of these scenarios, we extract the NP parameters simply by obtaining the weights with which the templates must be combined to reproduce the Monte-Carlo data.

Although the fit algorithm is based on a simple premise, there are two issues that must be taken into account. First, the construction of the observables requires distinguishing the decay products of the $t$ from those of the $\bar{t}$. However, the final state contains two $\bar{b}$ 's, which are indistinguishable, at least in some parts of phase space. We designate the $\bar{b}$ that yields the smaller value of $\left|m_{t}-\sqrt{\left(p_{\bar{b}}+p_{b}+p_{c}\right)^{2}}\right|$ as that having come from the $t$ decay. However, if both $\bar{b}$ 's in the event yield sufficiently small values of this quantity (less than $15 \Gamma_{t}$ ), then we consider them to be indistinguishable and exclude such events from the analysis. Second, the contribution to $t \bar{t}$ production from a $q \bar{q}$ initial state is not included in the analytical expressions. However, this must be taken into account as there are no known algorithms that can efficiently separate $t \bar{t}$ pairs coming from gluon fusion from those occuring due to $q \bar{q}$ annihilation.

In order to examine the prospects for detecting the presence of NP in $t \rightarrow$ $b \bar{b} c$, and for its identification, we perform further simulations of the distributions/correlations. The simulations are done for either short-term or long-term measurements at the LHC. For the short-term analysis we use $10^{4}$ events of the type $p p \rightarrow t \bar{t} \rightarrow(b \bar{b} c)\left(\bar{b} \ell^{-} \bar{\nu}_{\ell}\right)$. This is expected to be delivered by 2020-2021. For the long term we use $10^{5}$ events, which is projected by the year 2030 .

In the short term not all distributions/correlations may be measured, and what can be learned about the NP depends on what measurements have been made. In the presence of a sufficiently-large NP contribution to $t \rightarrow b \bar{b} c$, the shapes of 
the distributions/correlations can be significantly modified. Thus, NP in $t \rightarrow b \bar{b} c$ may be inferred by observing a clear difference between the shape of a measured distribution and its SM prediction. Even if there is no discernible difference in the shapes, it may still be possible to obtain information about NP contributions. Using the above algorithm with a slight modification, one can perform a fit to a single distribution. In this case, not all the individual $\hat{A}_{i}^{\sigma}$ 's are extracted, but rather certain combinations of the $\hat{A}_{i}^{\sigma}$ 's. We show that, even for a scenario in which the presence of NP does not induce a substantial change in the shape of the distribution, a fit may still yield statistically-significant evidence that NP is present. Finally, if all six distributions/correlations are measured, we can use the algorithm to perform a simultaneous fit on all the observables to extract $\operatorname{Re}\left(X_{L L}^{V}\right)$ and all the $\hat{A}_{i}^{\sigma}$ 's separately. In the examples studied, we find that the values of all NP parameters agree with their input values within $\pm 1.7 \sigma$. Although the errors are large, this provides an approximate determination of the values of the NP parameters. More importantly it allows us to infer that a non-zero NP contribution to $t \rightarrow b \bar{b} c$ exists.

In the long term, it is likely that all six distributions/correlations will be measured. Furthermore, the availability of larger statistics will lead to an improvement in the quality of the fits. We find that, with $10^{5}$ events, the best-fit values of all NP parameters differ from their input values by at most $1 \sigma$. Thus, if NP is present in $t \rightarrow b \bar{b} c$, the fit will allow the determination of its nature.

Acknowledgments: The authors wish to thank the MadGraph and FeynRules Teams for extensive discussions about MadGraph and FeynRules, respectively. PS would like to thank Georges Azuelos for helpful discussions about MadGraph. The authors also wish to thank S. Judge and J. Melendez for collaboration at an early stage of this work and J. Kiers and R. Rezvani for helpful discussions. This work was financially supported by NSERC of Canada (DL, PS). This work has been partially supported by ANPCyT under grant No. PICT-PRH 2009-0054 and by CONICET (AS). The work of KK was supported by the U.S. National Science Foundation under Grant PHY-1215785. KK also acknowledges sabbatical support from Taylor University.

[1] K. Kiers, P. Saha, A. Szynkman, D. London, S. Judge and J. Melendez, companion paper.

[2] V. M. Abazov et al. [D0 Collaboration], Phys. Rev. D 85, 091104 (2012) arXiv:1201.4156 [hep-ex]]; T. A. Aaltonen et al. [CDF Collaboration], Phys. Rev. Lett. 111, 202001 (2013) [arXiv:1308.4050 [hep-ex]].

[3] J. Alwall, M. Herquet, F. Maltoni, O. Mattelaer and T. Stelzer, JHEP 1106, 128 (2011) arXiv:1106.0522 [hep-ph]]; http://madgraph.hep.uiuc.edu/

[4] K. Kiers, T. Knighton, D. London, M. Russell, A. Szynkman and K. Webster, Phys. Rev. D 84, 074018 (2011) arXiv:1107.0754 [hep-ph]].

[5] A. Datta and D. London, Phys. Lett. B 595, 453 (2004) hep-ph/0404130. 
[6] E. Byckling and K. Kajantie, Particle Kinematics (Wiley, New York, 1973).

[7] A. Alloul, N. D. Christensen, C. Degrande, C. Duhr and B. Fuks, Comput. Phys. Commun. 185, 2250 (2014) arXiv:1310.1921 [hep-ph]]. http://feynrules.irmp.ucl.ac.be/.

[8] William Press et al., "Numerical Recipes in Fortran 77: The Art of Scientific Computing", Cambridge University Press, ISBN-13: 978-0521430647.

[9] N. Kidonakis, arXiv:1304.7775 [hep-ph]; S. Moch and P. Uwer, Phys. Rev. D 78, 034003 (2008) arXiv:0804.1476 [hep-ph]].

[10] Steve Myers, https://indico.cern.ch/event/73513/session/13/contribution/73 